\title{
C++ Quick Syntax Reference
}

Mikael Olsson 


\title{
C++ Quick Syntax Reference
}

\author{
Copyright (C) 2013 by Mikael Olsson
}

This work is subject to copyright. All rights are reserved by the Publisher, whether the whole or part of the material is concerned, specifically the rights of translation, reprinting, reuse of illustrations, recitation, broadcasting, reproduction on microfilms or in any other physical way, and transmission or information storage and retrieval, electronic adaptation, computer software, or by similar or dissimilar methodology now known or hereafter developed. Exempted from this legal reservation are brief excerpts in connection with reviews or scholarly analysis or material supplied specifically for the purpose of being entered and executed on a computer system, for exclusive use by the purchaser of the work. Duplication of this publication or parts thereof is permitted only under the provisions of the Copyright Law of the Publisher's location, in its current version, and permission for use must always be obtained from Springer. Permissions for use may be obtained through RightsLink at the Copyright Clearance Center. Violations are liable to prosecution under the respective Copyright Law.

ISBN-13 (pbk): 978-1-4302-6277-0

ISBN-13 (electronic): 978-1-4302-6276-7

Trademarked names, logos, and images may appear in this book. Rather than use a trademark symbol with every occurrence of a trademarked name, logo, or image we use the names, logos, and images only in an editorial fashion and to the benefit of the trademark owner, with no intention of infringement of the trademark.

The use in this publication of trade names, trademarks, service marks, and similar terms, even if they are not identified as such, is not to be taken as an expression of opinion as to whether or not they are subject to proprietary rights.

While the advice and information in this book are believed to be true and accurate at the date of publication, neither the authors nor the editors nor the publisher can accept any legal responsibility for any errors or omissions that may be made. The publisher makes no warranty, express or implied, with respect to the material contained herein.

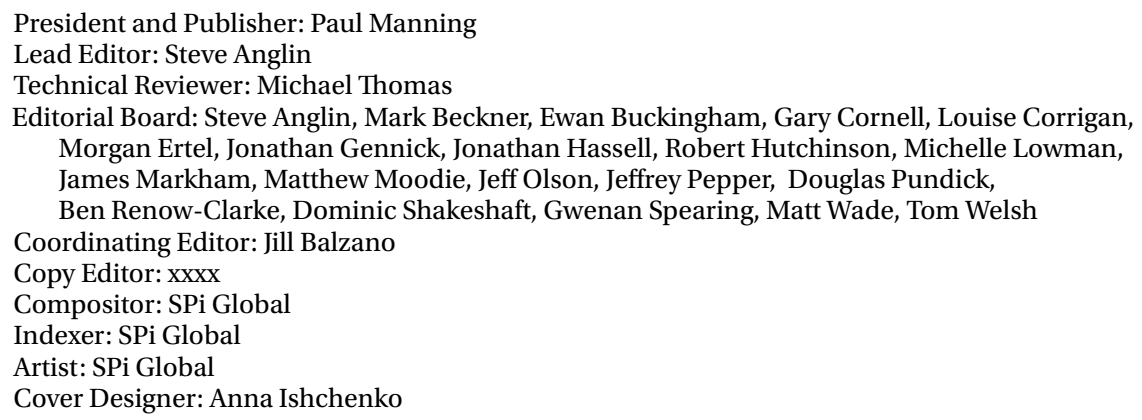

Distributed to the book trade worldwide by Springer Science+Business Media New York, 233 Spring Street, 6th Floor, New York, NY 10013. Phone 1-800-SPRINGER, fax (201) 348-4505, e-mail orders-ny@springer-sbm.com, or visit www.springeronline.com. Apress Media, LLC is a California LLC and the sole member (owner) is Springer Science + Business Media Finance Inc (SSBM Finance Inc). SSBM Finance Inc is a Delaware corporation.

For information on translations, please e-mail rights@apress.com, or visit wWw.apress.com.

Apress and friends of ED books may be purchased in bulk for academic, corporate, or promotional use. eBook versions and licenses are also available for most titles. For more information, reference our Special Bulk Sales-eBook Licensing web page at www. apress . com/bulk-sales.

Any source code or other supplementary material referenced by the author in this text is available to readers at WWW. apress. com. For detailed information about how to locate your book's source code, go to www. apress.com/source-code 


\section{Contents at a Glance}

About the Author xiii

About the Technical Reviewer ......................................................... XV

Introduction ................................................................................ Xvii

Chapter 1: Hello World.................................................................... 1

Chapter 2: Compile and Run ................................................................ 3

Chapter 3: Variables .................................................................. 5

Chapter 4: Operators ....................................................................11

Chapter 5: Pointers............................................................... 15

Chapter 6: References .................................................................... 19

Chapter 7: Arrays …..................................................................... 21

Chapter 8: String ................................................................ 23

Chapter 9: Conditionals ........................................................ 27

Chapter 10: Loops....................................................................... 29

Chapter 11: Functions ............................................................. 31

Chapter 12: Class .............................................................. 37

Chapter 13: Constructor ............................................................. 41

Chapter 14: Inheritance............................................................ 45

Chapter 15: Overriding ................................................................. 47

Chapter 16: Access Levels............................................................. 51 


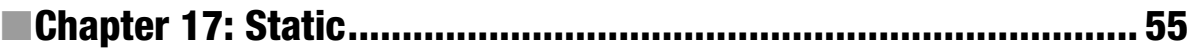

Chapter 18: Enum .................................................................. 57

Chapter 19: Struct and Union ................................................ 59

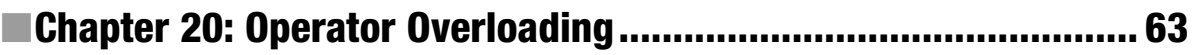

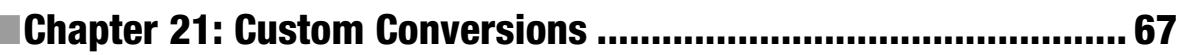

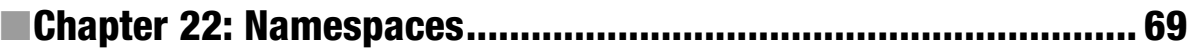

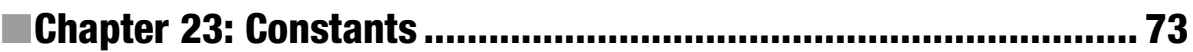

Chapter 24: Preprocessor.............................................................. 77

Chapter 25: Exception Handling ................................................. 83

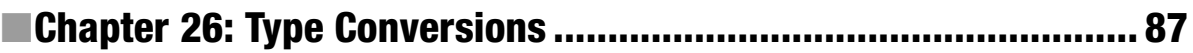

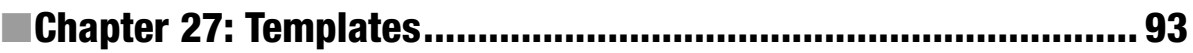

Chapter 28: Headers ........................................................................... 99

Index 


\section{Contents}

About the Author xiii

About the Technical Reviewer ........................................................ XV

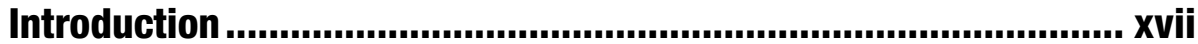

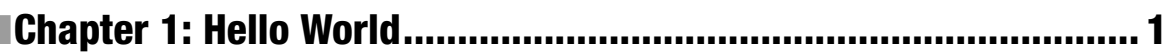

Choosing an IDE

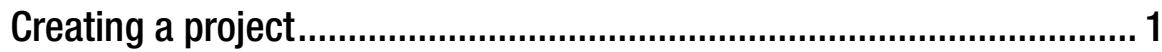

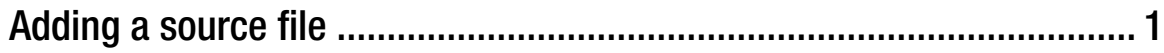

Hello world

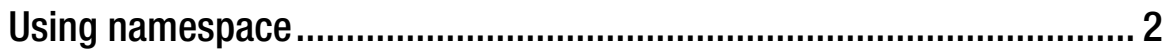

Chapter 2: Compile and Run ............................................................ 3

Visual Studio compilation....................................................................... 3

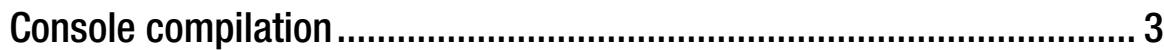

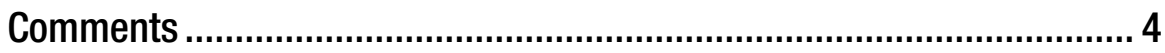

Chapter 3: Variables ..................................................................... 5

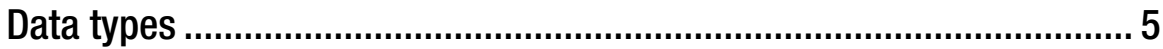

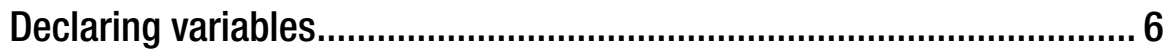

Assigning variables

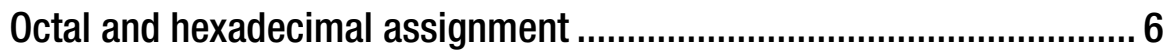

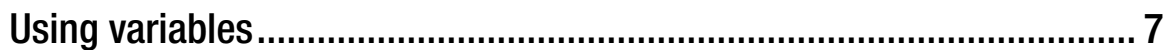

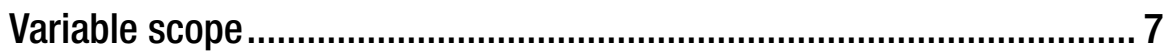

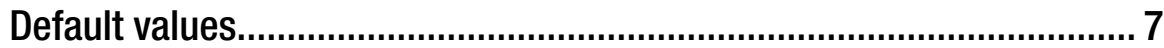


Integer types ...................................................................................... 7

Signed and unsigned integers............................................................... 8

Floating-point types ....................................................................... 9

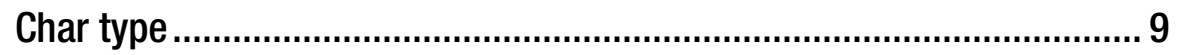

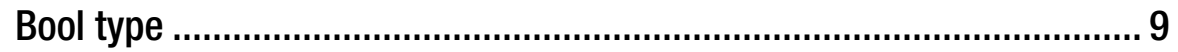

Chapter 4: Operators ......................................................... 11

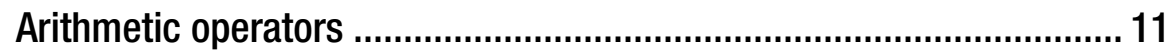

Assignment operators ................................................................... 11

Combined assignment operators ....................................................... 11

Increment and decrement operators.................................................... 12

Comparison operators ....................................................................... 12

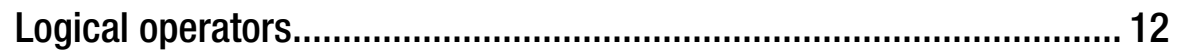

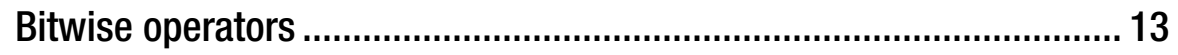

Operator precedence..................................................................... 13

Chapter 5: Pointers................................................................ 15

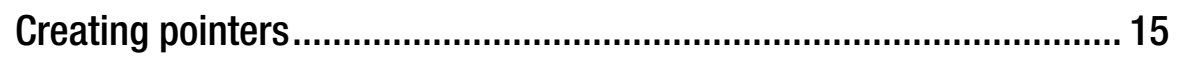

Dereferencing pointers.......................................................................... 15

Pointing to a pointer ............................................................................. 16

Dynamic allocation .......................................................................... 16

Null pointer..................................................................................... 17

Chapter 6: References ............................................................ 19

Creating references.......................................................................... 19

References and pointers .................................................................... 19

Reference and pointer guideline ....................................................... 19 


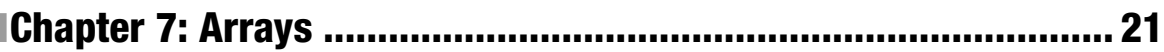

Array declaration and allocation ....................................................... 21

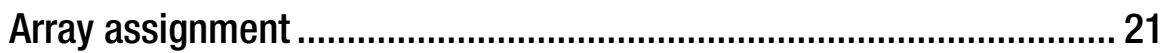

Multi-dimensional arrays ............................................................. 22

Dynamic arrays ....................................................................... 22

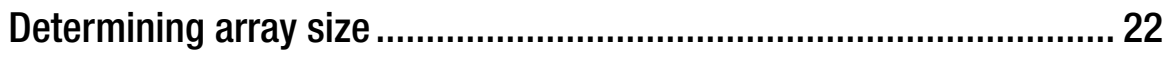

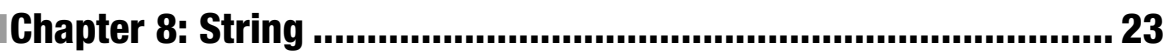

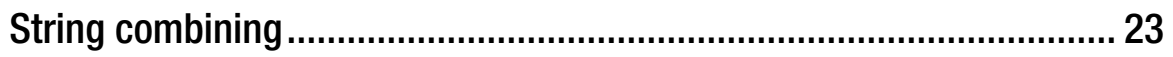

Escape characters..................................................................... 24

String compare................................................................... 24

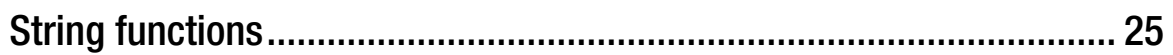

Chapter 9: Conditionals ............................................................. 27

If statement

Switch statement .................................................................... 28

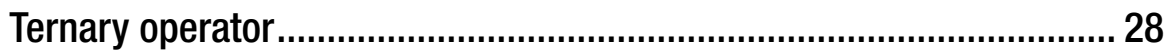

Chapter 10: Loops............................................................. 29

While loop

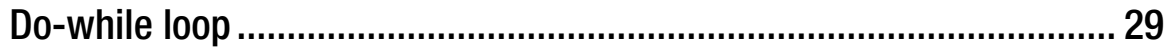

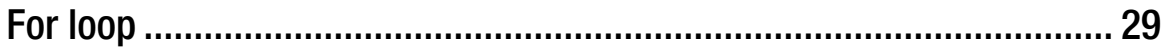

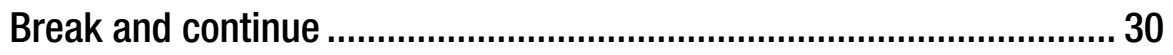

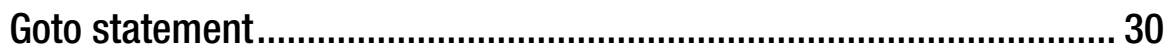

Chapter 11: Functions ......................................................... 31

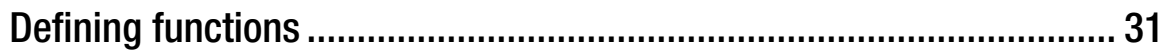

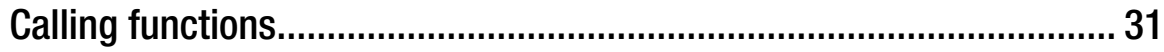

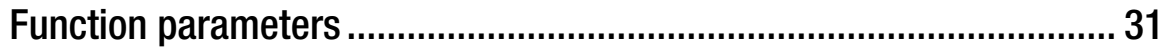

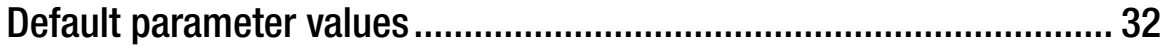


Function overloading......................................................................... 32

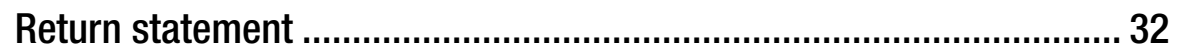

Forward declaration ...................................................................... 33

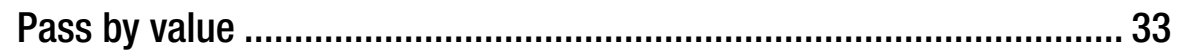

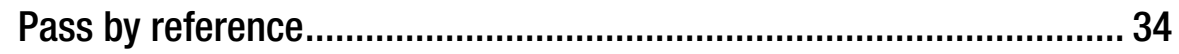

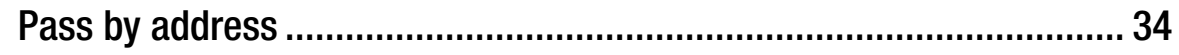

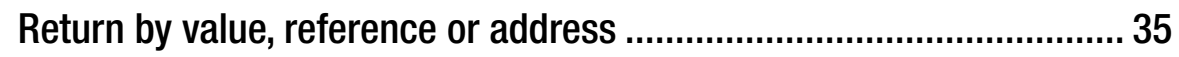

Inline functions.............................................................................. 36

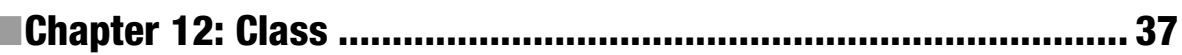

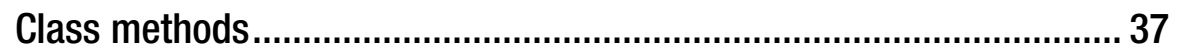

Inline methods................................................................................. 38

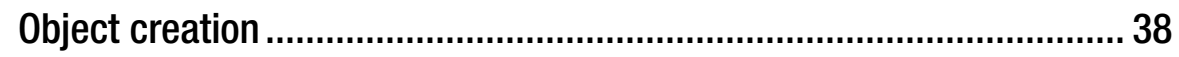

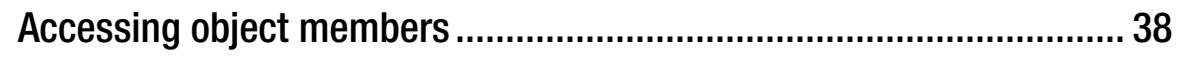

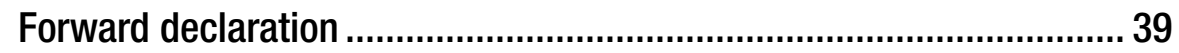

Chapter 13: Constructor ........................................................ 41

Constructor overloading .................................................................... 41

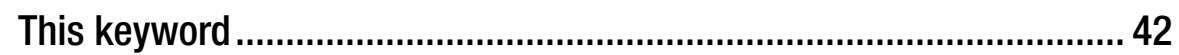

Constructor initialization list ............................................................. 42

Default constructor....................................................................... 42

Destructor .................................................................................... 43

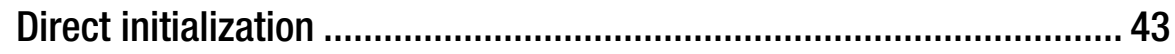

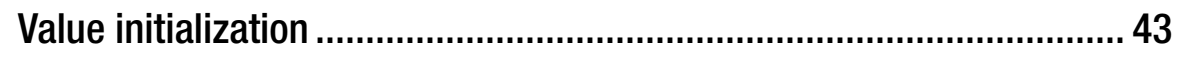

Copy initialization ............................................................................ 44

New initialization........................................................................... 44 
Chapter 14: Inheritance.............................................................. 45

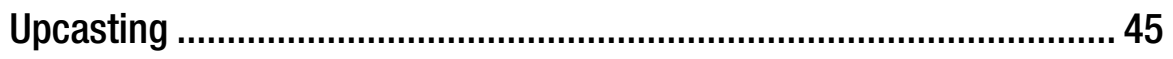

Downcasting............................................................................ 46

Multiple inheritance ..................................................................... 46

Chapter 15: Overriding ......................................................... 47

Hiding derived members ................................................................. 47

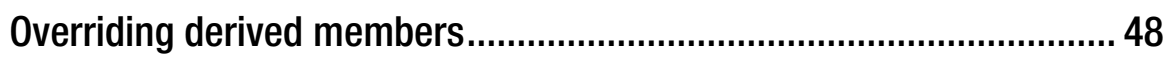

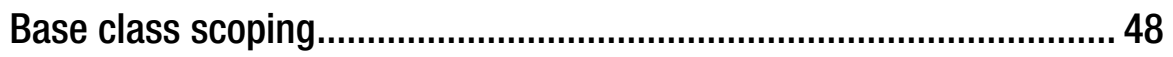

Calling base class constructor .......................................................... 49

Chapter 16: Access Levels..................................................... 51

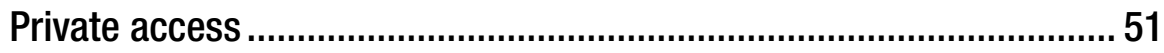

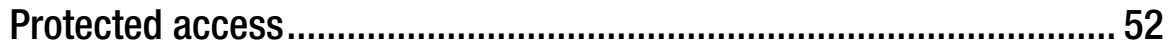

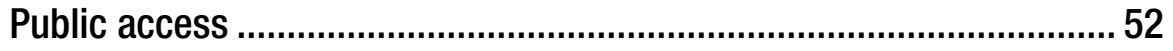

Access level guideline .................................................................. 52

Friend classes and functions ....................................................... 53

Public, protected and private inheritance............................................. 53

Chapter 17: Static................................................................. 55

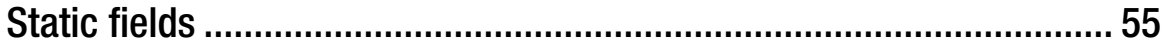

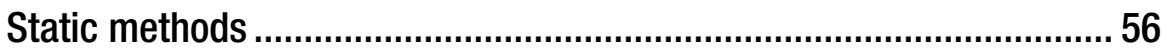

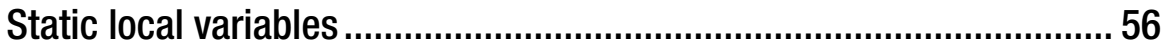

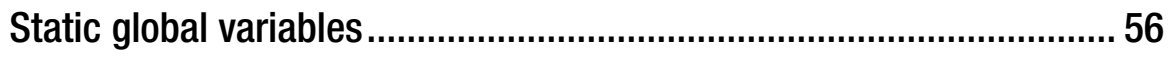

Chapter 18: Enum ................................................................... 57

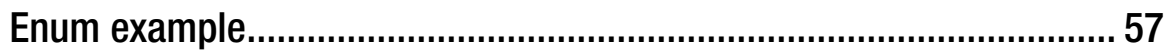

Enum constant values ........................................................... 58

Enum conversions .................................................................... 58

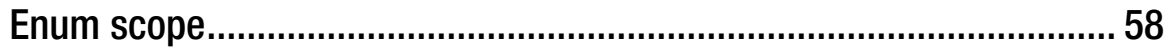


Chapter 19: Struct and Union .................................................... 59

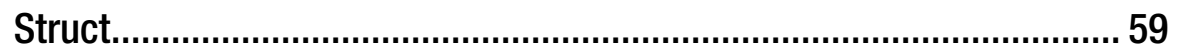

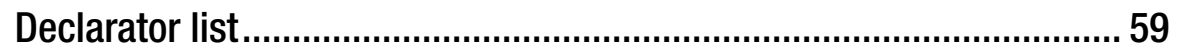

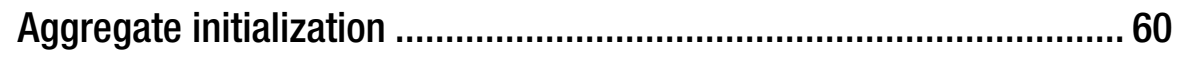

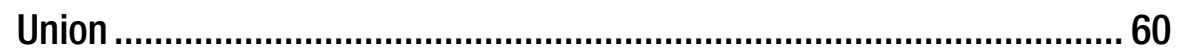

Anonymous union.......................................................................... 61

Chapter 20: Operator Overloading ........................................ 63

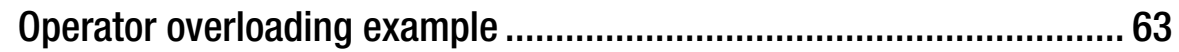

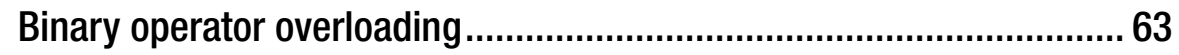

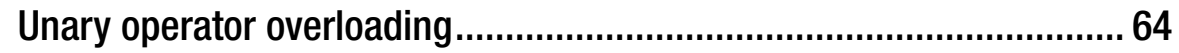

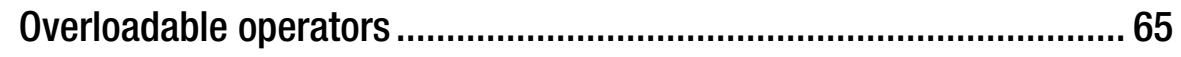

Chapter 21: Custom Conversions .............................................. 67

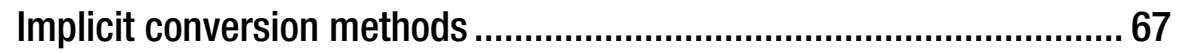

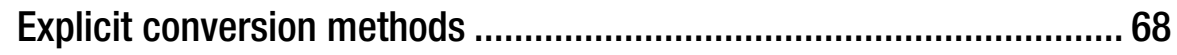

Chapter 22: Namespaces......................................................... 69

Accessing namespace members .................................................... 69

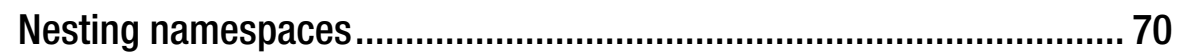

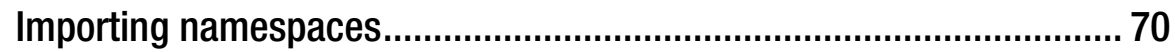

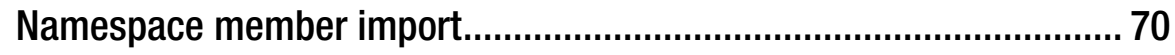

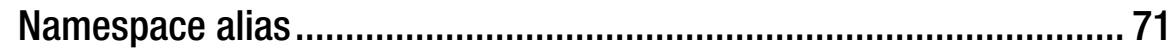

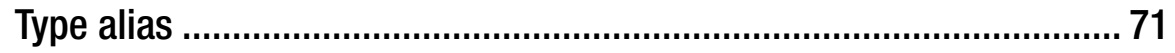

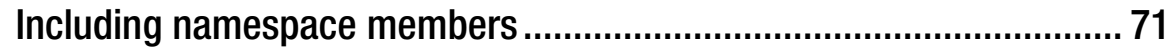

Chapter 23: Constants ........................................................... 73

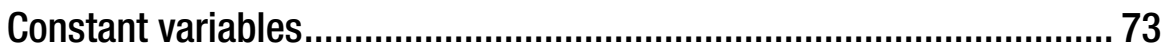

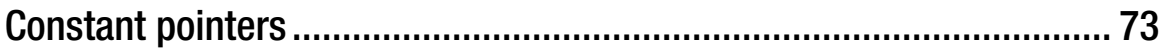

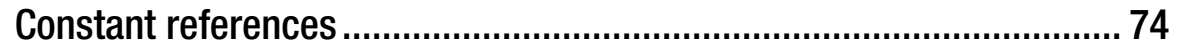

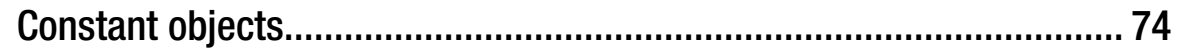




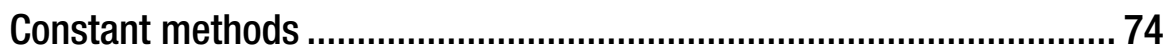

Constant return type and parameters ................................................ 75

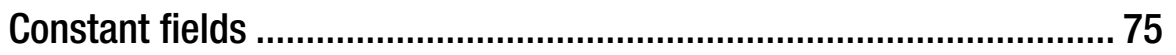

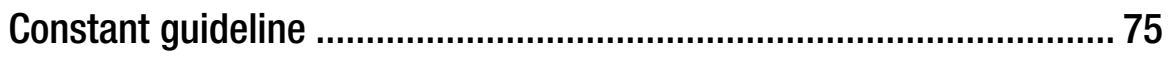

Chapter 24: Preprocessor.......................................................... 77

Including source files ................................................................ 77

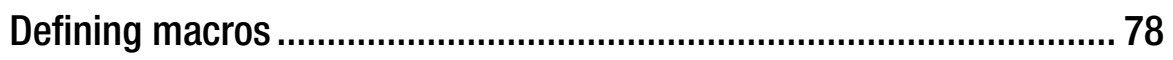

Undefining macros ................................................................. 78

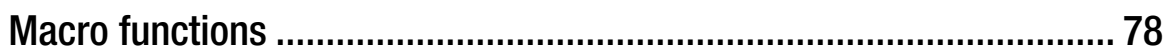

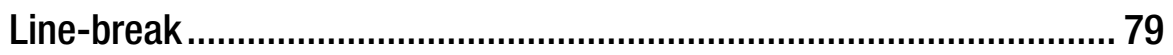

Avoid using macros .................................................................... 79

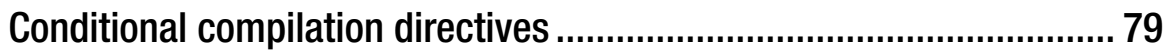

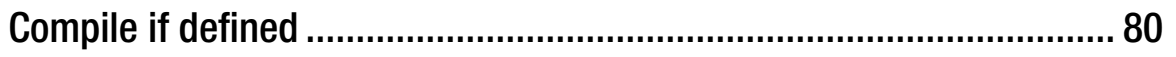

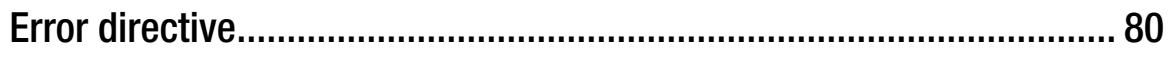

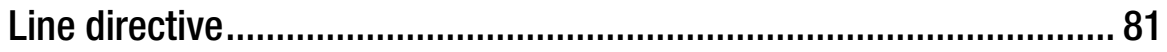

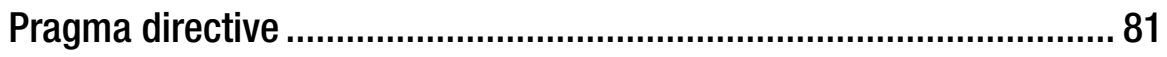

Chapter 25: Exception Handling ............................................. 83

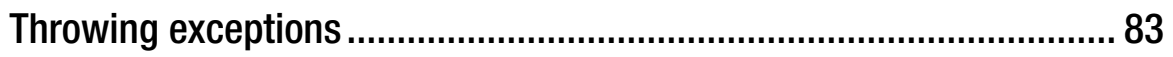

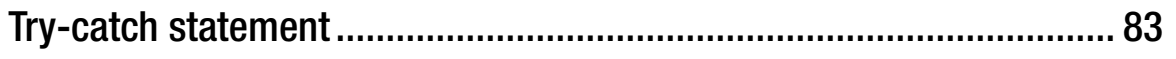

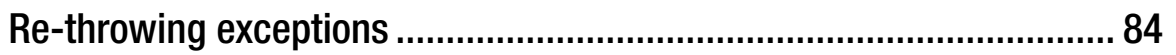

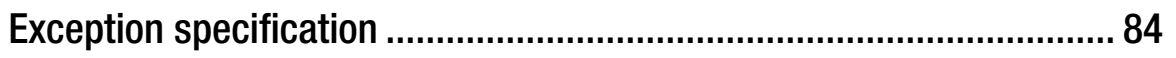

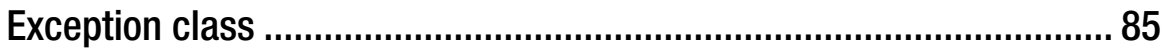

Chapter 26: Type Conversions .................................................... 87

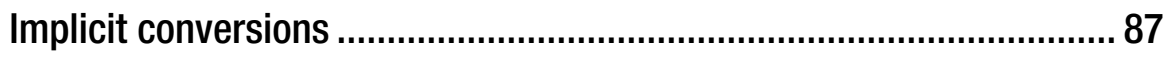

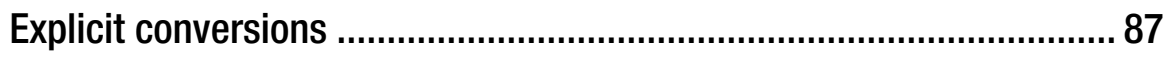

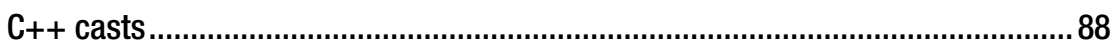




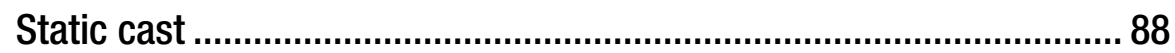

Reinterpret cast................................................................... 88

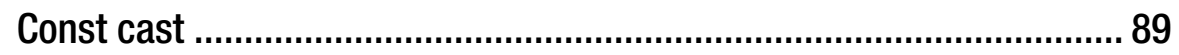

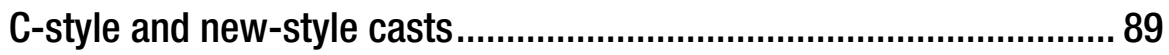

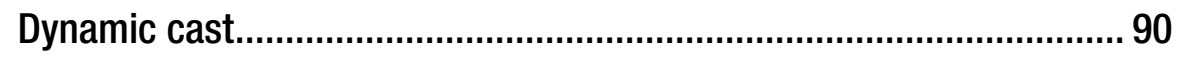

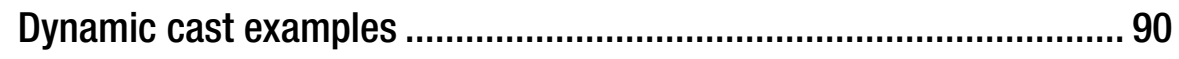

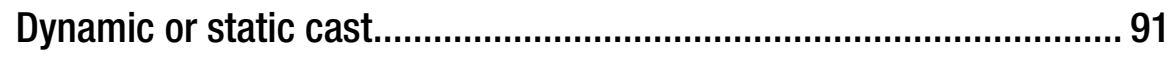

Chapter 27: Templates................................................................ 93

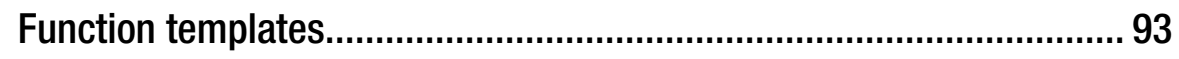

Calling function templates .............................................................. 94

Multiple template parameters......................................................... 94

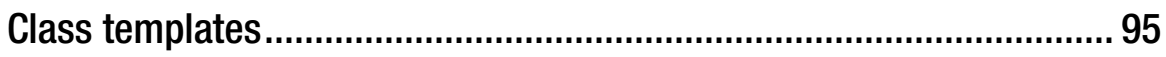

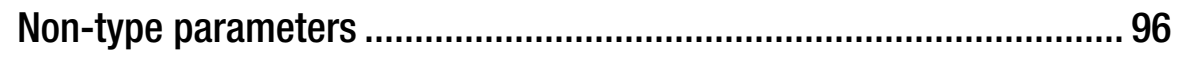

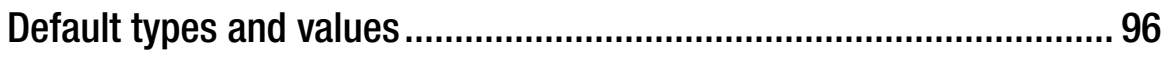

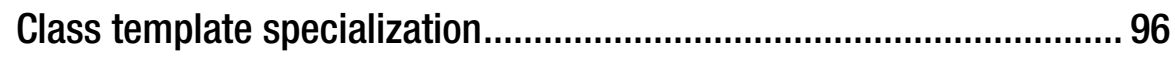

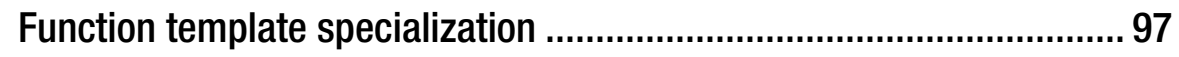

Chapter 28: Headers............................................................. 99

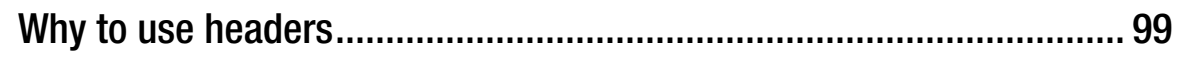

Using headers ......................................................................... 100

What to include in headers......................................................... 100

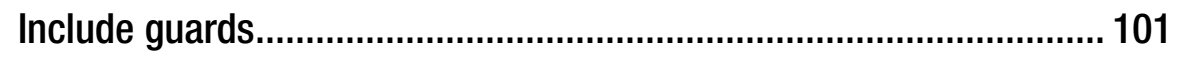

Index 


\section{About the Author}

Mikael Olsson is a professional web entrepreneur, programmer, and author. He works for an R\&D company in Finland where he specializes in software development.

In his spare time he writes books and creates websites that summarize various fields of interest. The books he writes are focused on teaching their subject in the most efficient way possible, by explaining only what is relevant and practical without any unnecessary repetition or theory. 



\section{About the Technical Reviewer}

Michael Thomas has worked in software development for over 20 years as an individual contributor, team lead, program manager, and Vice President of Engineering. Michael has over 10 years experience working with mobile devices. His current focus is in the medical sector using mobile devices to accelerate information transfer between patients and health care providers. 



\section{Introduction}

$\mathrm{C}++$ is a general purpose multi-paradigm programming language. It is an extension of the $\mathrm{C}$ language and as such most $\mathrm{C}$ code can easily be made to compile in $\mathrm{C}++$. Some of the major additions to $\mathrm{C}$ include object-orientated programming, operator overloading, multiple inheritance and exception handling.

The development of $\mathrm{C}++$ began in 1979, seven years after $\mathrm{C}$ first made its appearance. Despite being what many consider legacy languages, $\mathrm{C}$ and $\mathrm{C}++$ are still the most widely used languages in the software industry. They are used in creating everything from operating systems and embedded software to desktop applications, games and so on.

Compared with newer languages, $\mathrm{C}++$ applications are often more complex and take longer to develop. In return, $\mathrm{C}++$ gives the programmer a tremendous amount of control in that the language provides both high-level and low-level abstractions from the hardware. It is also designed to give the programmer a lot of freedom by supporting many different programming styles or paradigms, such as procedural, object-oriented or generic programming.

The compiler used in this book is the Microsoft $\mathrm{C}++$ compiler. Some other common ones include Borland, Intel and GNU C++ compilers. Despite $\mathrm{C}++$ being standardized in 1998, these compilers still support slightly different features. Therefore, when something applies specifically to the Microsoft compiler this will be pointed out. 